\title{
Relevance between Schmorl's Node and Lumbar Intervertebral Disc Degeneration Quantified with Magnetic Resonance Imaging T2 Mapping in Chronic Low Back Pain
}

\author{
Izaya Ogon ${ }^{1}$, Hiroyuki Takashima ${ }^{1}$, Tomonori Morita ${ }^{1}$, Tsutomu Oshigiri ${ }^{1}$, \\ Yoshinori Terashima ${ }^{1}$, Mitsunori Yoshimoto ${ }^{1}$, Ryunosuke Fukushi ${ }^{1}$, Shutaro Fujimoto ${ }^{1}$, \\ Makoto Emori ${ }^{1}$, Atsushi Teramoto ${ }^{1}$, Tsuneo Takebayashi ${ }^{2}$, Toshihiko Yamashita ${ }^{1}$ \\ ${ }^{1}$ Department of Orthopaedic Surgery, Sapporo Medical University School of Medicine, Sapporo, Japan \\ ${ }^{2}$ Department of Orthopaedic Surgery, Sapporo Maruyama Orthopaedic Hospital, Sapporo, Japan
}

Study Design: Cross-sectional study.

Purpose: The purpose of this study was to elucidate the relevance among Schmorl's node (SN), chronic low back pain (CLBP), and intervertebral disc degeneration (IVDD) with the use of magnetic resonance imaging T2 mapping.

Overview of Literature: SN may be combined with CLBP and/or IVDD; however, their relationship has not been determined to date. Methods: A total of 105 subjects were included (48 men and 57 women; mean age, 63.2 \pm 2.7 years; range, 22-84 years). We analyzed five functional spinal unit levels (L1-S1) and evaluated the T2 values of the anterior annulus fibrosus (AF), nucleus pulposus, and posterior AF. We compared the low back pain (LBP) Visual Analog Scale (VAS) scores and the T2 values in each decade with or without SN.

Results: There were no remarkable differences in SN prevalence rate regarding age decade or gender. SNs were more prevalent in the upper 2 levels (70.3\%). LBP VAS scores with and without SN were $64.7 \pm 4.3 \mathrm{~mm}$ and $61.9 \pm 2.8 \mathrm{~mm}$, respectively, with no significant differences between the groups $(p=0.62)$. The T2 values of anterior AF with $S N$ were significantly lower than those without $S N$ in patients in their 50s, 60s, 70s, and 80s $(p<0.01)$.

Conclusions: SN presence is not itself a risk factor for CLBP; however, it indicates IVDD of the anterior AF in subjects with SN who are $\geq 50$ years old.

Keywords: Low back pains; Intervertebral disc degeneration; Magnetic resonance imaging

\section{Introduction}

Low back pain (LBP) is a commonly observed condition and one of the most serious physiological issues world- wide [1]. It can be caused by a variety of problems in any part of the complex, such as the interconnected network of the spinal muscles, bones, discs, nerves, or tendons in the lumbar spine. Intervertebral disc degeneration (IVDD)

\footnotetext{
Received Jul 21, 2019; Revised Nov 2, 2019; Accepted Nov 28, 2019

Corresponding author: Izaya Ogon

Department of Orthopaedic Surgery, Sapporo Medical University School of Medicine, 291, Soutn-1, West-16, Chuo-ku, Sapporo, 0608543, Japan

Tel: +81-11-611-2111, Fax: +81-11-641-6026, E-mail: ogon.izaya@sapmed.ac.jp
} 
is considered to be the main cause of LBP [2]. Many studies have reported the pathology, diagnosis, and treatment of discogenic LBP; however, the underlying mechanism and treatment remain unclear.

Schmorl's node ( $\mathrm{SN}$ ) is an intraspongious disc herniation formed within the vertebral body through a cartilaginous endplate [3]. Although SNs are common in the spine [4], they are seldom symptomatic, and their clinical significance as an origin of LBP has not been determined. Nonetheless, a previous report has shown that SN might be an active symptomatic process and cause LBP in patients with evidence of inflammation and/or edema in the vertebral bone marrow [5]. The pathophysiology of the symptomatic cases and the radiological differences between the asymptomatic and symptomatic individuals are also controversial. Most SNs are reportedly combined with IVDD [6]; however, the relationship between SN and IVDD has not been examined or understood $[4,6]$.

Magnetic resonance imaging (MRI) is an important modality for intervertebral disc (IVD) diagnosis. Signal variation of the discs on T2-weighted images reflects the age and degeneration enabling disc degeneration determination. In particular, because MRI signal strength is related to water and proteoglycan content, changes in the MRI signal strength in the nucleus pulposus (NP) can indicate IVDD [7]. IVDD has been classified with the use of T2-weighted images, as described by Pfirrmann et al. [8]. Nonetheless, this strategy is based on visual evaluation; therefore, the quantification of degeneration is unclear. In recent years, there have been several studies that attempted to quantify lumbar disc degeneration with the use of MRI T2 and MRI T1p mapping [9-13]. MRI T2 mapping utilizes T2 relaxation time for quantification of moisture contents and collagen sequence breakdown. In our previous work, we used MRI T2 mapping to quantify the extent of IVDD and found a correlation with Pfirrmann classification [14]. Furthermore, we quantitatively evaluated IVDD with MRI T2 mapping and found a correlation between posterior annulus fibrosus (AF) degeneration and chronic low back pain (CLBP) [15].

The purpose of this study was to determine the connection among CLBP, SN, and IVDD, quantified with the use of MRI T2 mapping.

\section{Materials and Methods}

The institutional review board of the Sapporo Medical
University approved this study (IRB approval no., 2621074). All the subjects were presented with written and verbal explanations of the study, and they provided their consent before participation.

\section{Participants}

The standard Visual Analog Scale (VAS) scores to diagnose patients with LBP were not clearly defined. Nonetheless, there was a report that included patients with LBP whose VAS scores were more than $30 \mathrm{~mm}$; therefore, in this study, we marked out those whose VAS scores were more than $30 \mathrm{~mm}$. The exclusion criteria included the following: (1) systemic inflammatory disease; (2) neurological disorder; (3) previous spine surgery; (4) neoplasm, infection, or acute trauma; and (5) spondylolisthesis and scoliosis. There were a total of 105 patients (48 men and 57 women; mean age, $63.2 \pm 2.7$ years; range, $22-84$ years). We analyzed the five functional spinal unit levels (L1-L2, L2-L3, L3-L4, L4-L5, and L5-S1), obtaining a total of 725 levels. All the subjects completed the LBP VAS (scores 0-100 $\mathrm{mm}$ ) and underwent MRI of the lumbar spine. We also calculated the body mass index (BMI) with the use of the self-reported body weight $(\mathrm{kg})$ divided by the height squared $\left(\mathrm{m}^{2}\right)$.

\section{Magnetic resonance imaging protocol}

The equipment used consisted of a spine coil with a GE Signa HDx 1.5 T (GE Healthcare, Milwaukee, WI, USA). T2-weighted sagittal images (repetition time [TR], 4,000 ms; echo time [TE], $102 \mathrm{~ms}$; receive band width [RBW], $\pm 31.25 \mathrm{kHz}$; field of view [FOV], $24 \mathrm{~cm}$; matrix, $384 \times 288$; slice thickness/gap, $4 \mathrm{~mm} / 1 \mathrm{~mm}$; number of excitations [NEX], 4; and total scan time, 3 minutes and 4 seconds) were obtained.

\section{Assessment of Schmorl's node}

SN was defined as a localized defect at the cranium, caudal endplate, or both endplates, with a well-defined herniation that concaved in the vertebral body, with or without a surrounding sclerotic rim with low signal intensity (SI) on T2-weighted image [16]. We did not consider the erosive defects of the endplate at the degenerate segments as $\mathrm{SN}$. For the intra- and interobserver reliabilities, two investigators (observer 1, I.O. and observer 2, H.T.) scored 


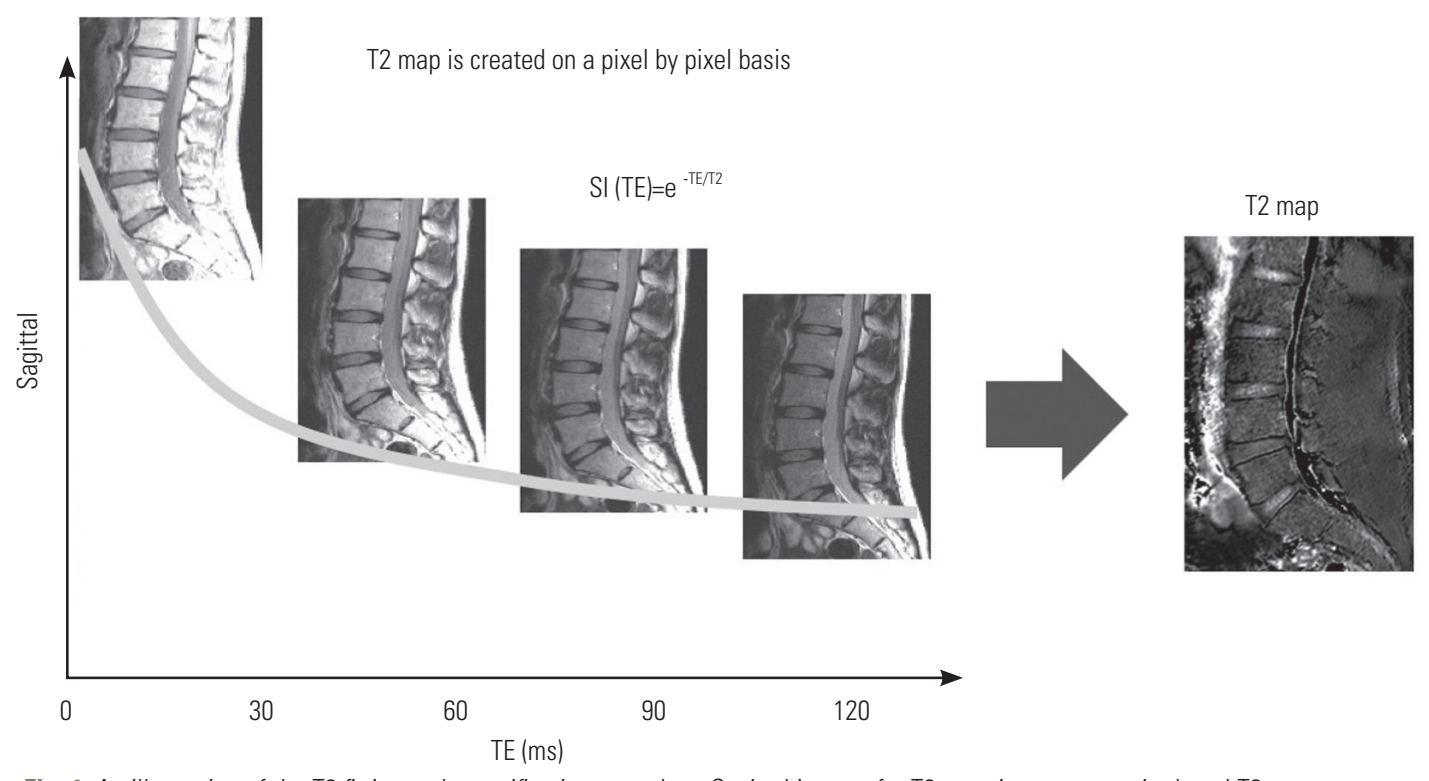

Fig. 1. An illustration of the T2 fitting and quantification procedure. Sagittal images for T2 mapping were acquired, and T2 maps were created on a pixel-by-pixel basis. SI, signal intensity; TE, echo time.

the magnetic resonance (MR) images in the same manner. The intra- and interobserver variabilities for $\mathrm{SN}$ were 0.93 and 0.90 , respectively.

\section{Magnetic resonance imaging T2 mapping}

The sagittal images for T2 mapping were acquired, and T2 maps were created on a pixel-by-pixel basis (Fig. 1). We used the T2 values in the midsagittal section, the sagittal sections centered on the lumbar midline region with optimized 8 echo multispin echo (TR/first echo TE, last echo TE, 1,000/14.8, 118.6; RBW, $\pm 15.63 \mathrm{kHz}$; FOV, $22 \mathrm{~cm}$; matrix, $320 \times 256$; slice thickness/gap, $4 \mathrm{~mm} / 4 \mathrm{~mm} ; 5$ slices; NEX, 2; and total scan time, 8 minutes and 34 seconds) obtained with an Advantage Workstation (ver. 4.4, Functool; GE Healthcare, Milwaukee, WA, USA). Nevertheless, the first echo from the multispin system was excluded to minimize the effect of the stimulated echo. T2 map was calculated in each pixel from the SI in the respective TE with the following formula: $\mathrm{SI}(\mathrm{TE})=\mathrm{e}^{-\mathrm{TE} / \mathrm{T} 2}$.

To measure the disc, it was divided into five equal areas that designate the front fifth of the anterior AF, the middle fifth of the NP, and the last fifth of the posterior AF [14,15], at five functional spinal unit levels (L1-L2, L2-L3, L3-L4, L4-L5, and L5-S1) (Fig. 2). In the same region, we measured the mean values obtaining a total of 725 levels. The $\mathrm{T} 2$ values were measured by an individual with $\mathrm{PhD}$ degree (H.T. who has 12-year experience in spine MR image

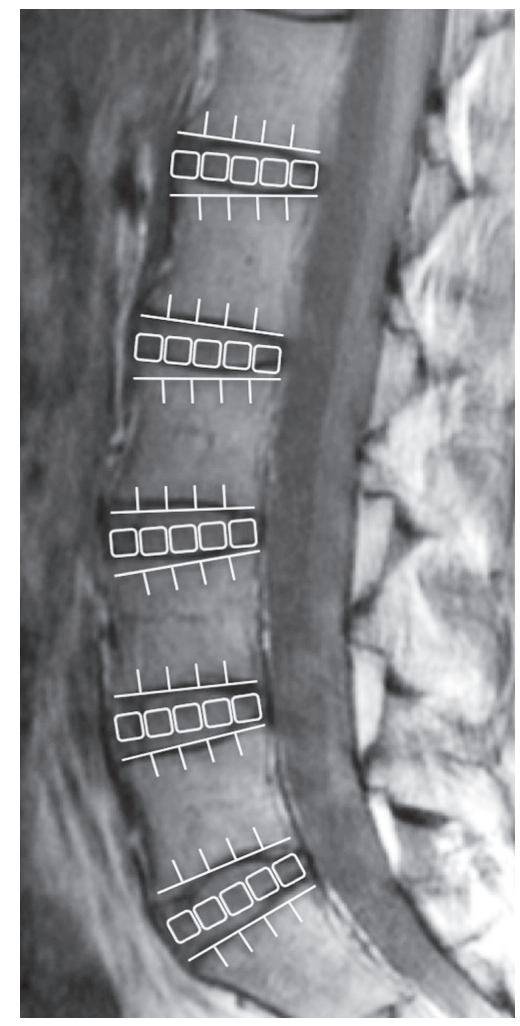

Fig. 2. In the second echo image, the disc was divided into five equal areas, which designated the front fifth of the anterior $\mathrm{AF}$, the middle fifth of the nucleus pulposus, and the last fifth of the posterior $A F$, at five functional spinal unit levels (L1-L2, L2-L3, L3-L4, L4-L5, and L5-S1). AF, annulus fibrosus.

analysis) with the use of MedCalc (ver. 10.2.0.0; MedCalc Software, Mariakerke, Belgium). 


\section{Statistical analyses}

The subjects were divided into the following five groups based on their age: $20 \mathrm{~s}, 30 \mathrm{~s}, 40 \mathrm{~s}, 50 \mathrm{~s}, 60 \mathrm{~s}, 70 \mathrm{~s}$, and $80 \mathrm{~s}$ (more than 10 subjects in each age decade). The SN prevalence in each decade and gender was analyzed with the use of the chi-square test, and the SN distribution level was examined. We compared the LBP VAS scores and the T2 values with or without SN with the use of the MannWhitney $U$-test. A probability value of $<0.05$ indicated statistical significance. All the numerical data are expressed as the means \pm standard error of the mean values.

\section{Results}

The patients were categorized into two groups, with SN $(\mathrm{n}=32)$ and without $\mathrm{SN}(\mathrm{n}=73)$ (Table 1$)$. The study population composed of $30.5 \%$ with those with $\mathrm{SN}(\mathrm{n}=32)$, of which 13 were men (40.6\%) and 19 were women (59.4\%), and $69.5 \%$ with those without $\mathrm{SN}(\mathrm{n}=73)$, of which 35 were men $(47.9 \%)$ and 38 were women $(52.1 \%)$. Nevertheless, there were no statistically significant gender differences $(p=0.49)$. The mean age of the subjects with SN and without $\mathrm{SN}$ was $63.9 \pm 2.8$ years and $63.1 \pm 2.2$ years, respectively, but this difference was not statistically significant $(p=0.72)$. The mean BMI of the subjects with SN and without SN was $23.4 \pm 0.5 \mathrm{~kg} / \mathrm{m}^{2}$ and $23.9 \pm 0.6 \mathrm{~kg} / \mathrm{m}^{2}$, respectively, but this difference was also not statistically significant $(p=0.79)$. Furthermore, the LBP VAS scores in those with and without SN were $64.7 \pm 2.1 \mathrm{~mm}$ and $61.9 \pm 2.8 \mathrm{~mm}$, respectively, with no significant differences between the groups $(p=0.62)$. The number of patients with SN per the age decade was as follows: three out of 10 for the $20 \mathrm{~s}$, three out of 10 for the $30 \mathrm{~s}$, four out of 14 for the 40 s, five out of 16 for the 50s, six out of 19 for the 60 s,

Table 1. Patient demographic data concerning the gender, age, BMI, and VAS score

\begin{tabular}{lccc} 
Characteristic & $\begin{array}{c}\text { With SN } \\
(\mathrm{n}=32)\end{array}$ & $\begin{array}{c}\text { Without SN } \\
(\mathrm{n}=73)\end{array}$ & $p$-value \\
\hline Gender (male:female) & $13: 19$ & $35: 38$ & $0.49^{\mathrm{a})}$ \\
\hline Age $(\mathrm{yr})$ & $63.9 \pm 2.8$ & $63.1 \pm 2.2$ & $0.72^{\mathrm{b})}$ \\
BMI $\left(\mathrm{kg} / \mathrm{m}^{2}\right)$ & $23.4 \pm 0.5$ & $23.9 \pm 0.6$ & $0.79^{\mathrm{b})}$ \\
VAS score $(\mathrm{mm})$ & $64.7 \pm 2.1$ & $61.9 \pm 2.8$ & $0.62^{\mathrm{b})}$ \\
\hline
\end{tabular}

Values are presented as number or mean \pm standard error of the mean. BMI, body mass index; VAS, Visual Analog Scale; SN, Schmorl's node. alBy chi-square test. ${ }^{b / B y}$ Mann-Whitney U-test. seven out of 22 for the 70 s, four out of 14 for the 80 s, and 32 out of 105 for the total study population (Fig. 3). There were no remarkable differences in the $\mathrm{SN}$ prevalence rate regarding the age decade. In those with $\mathrm{SN}, 87.5 \%(\mathrm{n}=28)$ had single, 9.4\% $(n=3)$ double, and 3.1\% $(n=3)$ triple lumbar-level involvement. The SN distribution was $32.4 \%$ (12 out of 37) for the L1-2 level, 37.9\% (14 out of 37) for L2-3, 16.2\% (six out of 37) for L3-4 level, 10.8\% (four out of 37) for L4-5 level, and 2.7\% (one out of 37) for L5$\mathrm{S}$ (Fig. 4). SNs were more prevalent in the upper 2 levels (70.3\%), especially in L2/3.

The T2 values of the anterior AF with and without SN were as follows, respectively: $84.5 \pm 6.4 \mathrm{~ms}$ and $85.6 \pm 7.9$ $\mathrm{ms}$ in the $20 \mathrm{~s} ; 86.8 \pm 4.3 \mathrm{~ms}$ and $83.4 \pm 8.4 \mathrm{~ms}$ in the $30 \mathrm{~s}$; $77.6 \pm 6.5 \mathrm{~ms}$ and $80.8 \pm 8.6 \mathrm{~ms}$ in the $40 \mathrm{~s} ; 65.2 \pm 3.4 \mathrm{~ms}$ and $76.4 \pm 4.3 \mathrm{~ms}$ in the $50 \mathrm{~s} ; 50.8 \pm 2.6 \mathrm{~ms}$ and $64.1 \pm 4.7 \mathrm{~ms}$ in the $60 \mathrm{~s} ; 49.3 \pm 3.5 \mathrm{~ms}$ and $65.7 \pm 4.1 \mathrm{~ms}$ in the $70 \mathrm{~s}$; and $42.5 \pm 4.2 \mathrm{~ms}$ and $54.7 \pm 3.6 \mathrm{~ms}$ in the 80 s (Fig. 5). There

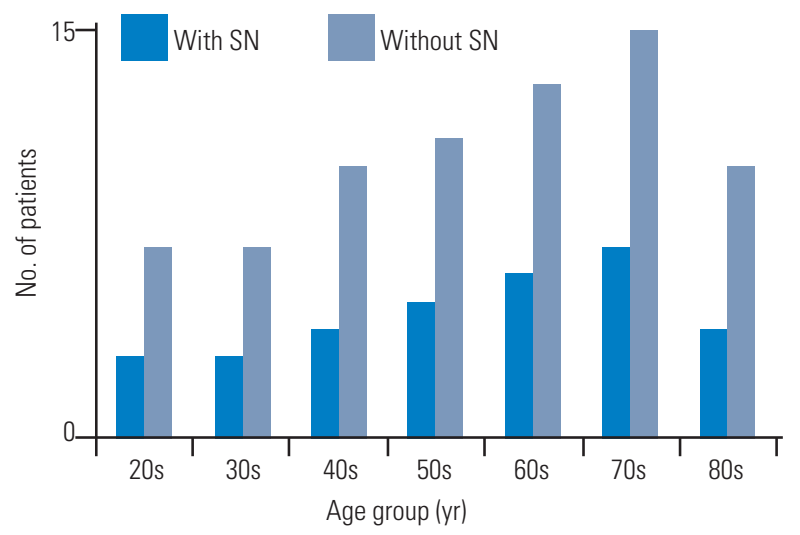

Fig. 3. Bar chart of the number of patients with and without SNs in each age decade. There were no remarkable differences in SN prevalence rate regarding age decade. SN, Schmorl's node.

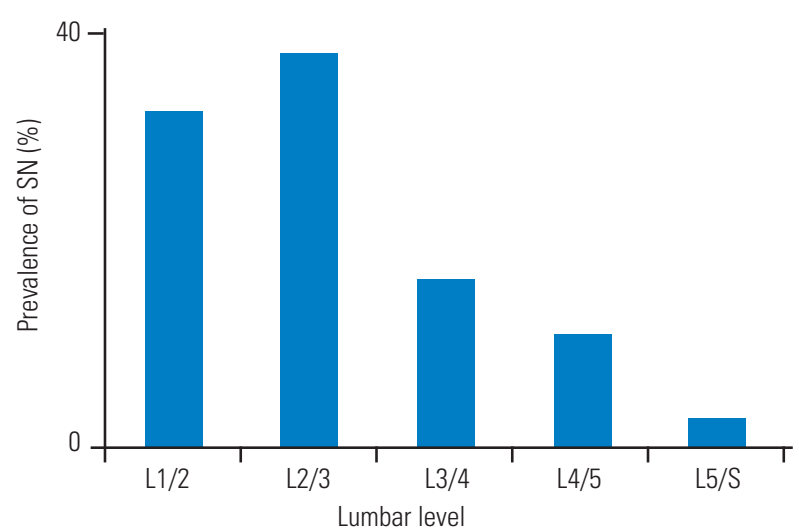

Fig. 4. Bar chart of the overall percent prevalence of SN as per the lumbar level. SNs were more prevalent in the upper 2 levels, and L2/3 was the most common level. SN, Schmorl's node. 
were no significant differences in the T2 values of anterior AF with and without SN in patients in their 20s, 30s, and $40 \mathrm{~s}$ ( $p=0.75,0.60$, and 0.78 , respectively). Nonetheless, the T2 values of anterior AF with $\mathrm{SN}$ were significantly lower than those without $\mathrm{SN}$ in patients in their $50 \mathrm{~s}, 60 \mathrm{~s}$, 70 s, and 80 s $(p<0.01)$. Furthermore, the T2 values of the
NP with and without SN were as follows, respectively: $94.7 \pm 5.0 \mathrm{~ms}$ and $90.8 \pm 6.9 \mathrm{~ms}$ in the $20 \mathrm{~s} ; 90.0 \pm 6.3 \mathrm{~ms}$ and $92.5 \pm 4.1 \mathrm{~ms}$ in the $30 \mathrm{~s} ; 80.8 \pm 7.5 \mathrm{~ms}$ and $80.1 \pm 5.8 \mathrm{~ms}$ in the $40 \mathrm{~s} ; 78.5 \pm 8.1 \mathrm{~ms}$ and $80.3 \pm 4.3 \mathrm{~ms}$ in the $50 \mathrm{~s} ; 67.8 \pm 3.8$ $\mathrm{ms}$ and $68.3 \pm 4.5 \mathrm{~ms}$ in the $60 \mathrm{~s} ; 64.8 \pm 5.1 \mathrm{~ms}$ and $68.0 \pm 3.8$ $\mathrm{ms}$ in the $70 \mathrm{~s}$; and $60.0 \pm 4.2 \mathrm{~ms}$ and $60.8 \pm 3.9 \mathrm{~ms}$ in the

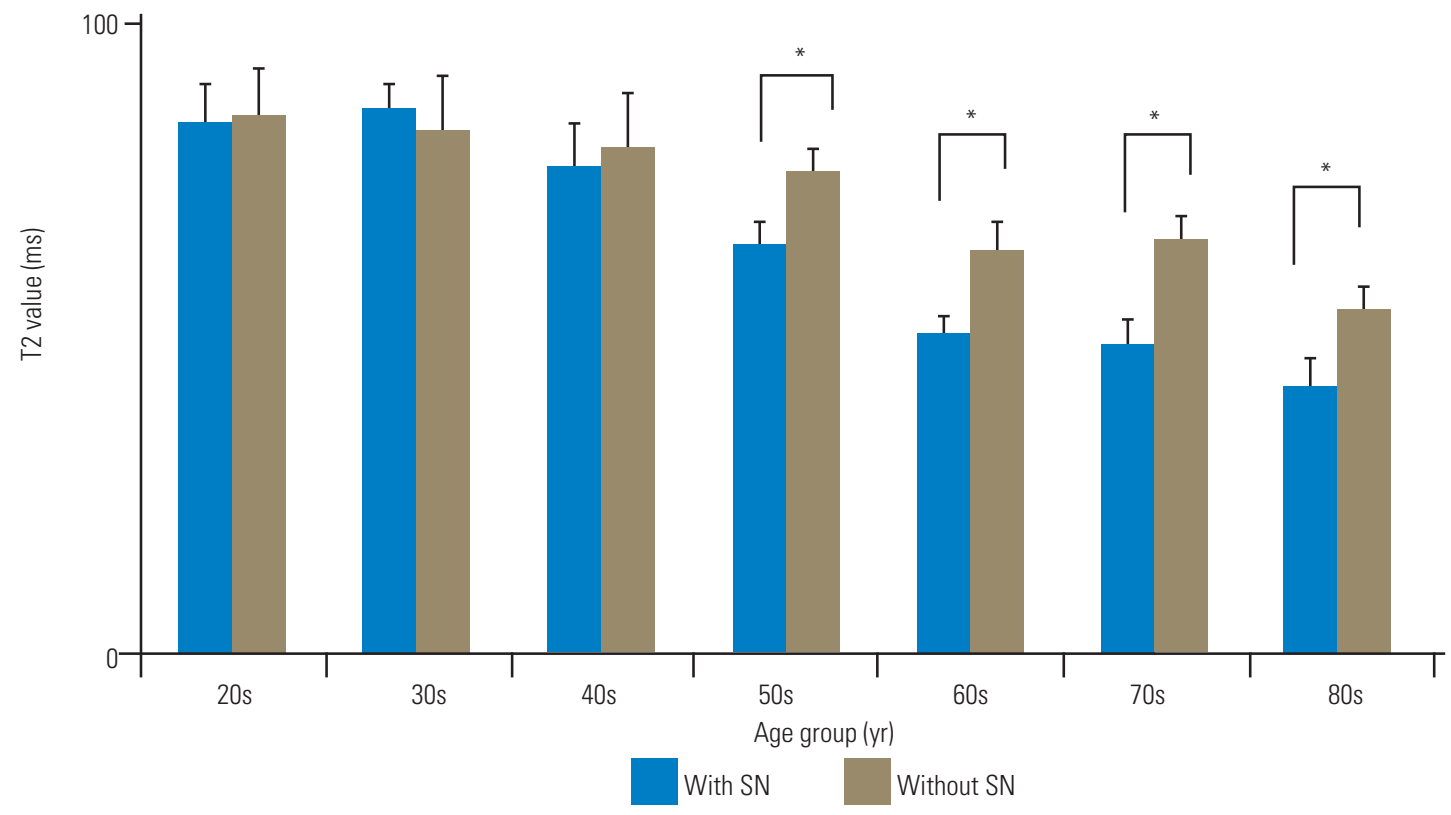

Fig. 5. Bar chart of T2 values with and without SN as anterior AF. There were no significant differences in the T2 values of anterior AF with and without $S N$ in patients in their 20s, 30s, and $40 \mathrm{~s}(p=0.75,0.60$, and 0.78 , respectively). The T2 values of anterior AF with $S N$ were remarkably lower than those without SN in patients in their 50s, 60s, 70s, and 80s. Error bars denote the standard error of the mean values. SN, Schmorl's node; $A F$, annulus fibrosus. ${ }^{*} p<0.01$ (by Mann-Whitney U-test).

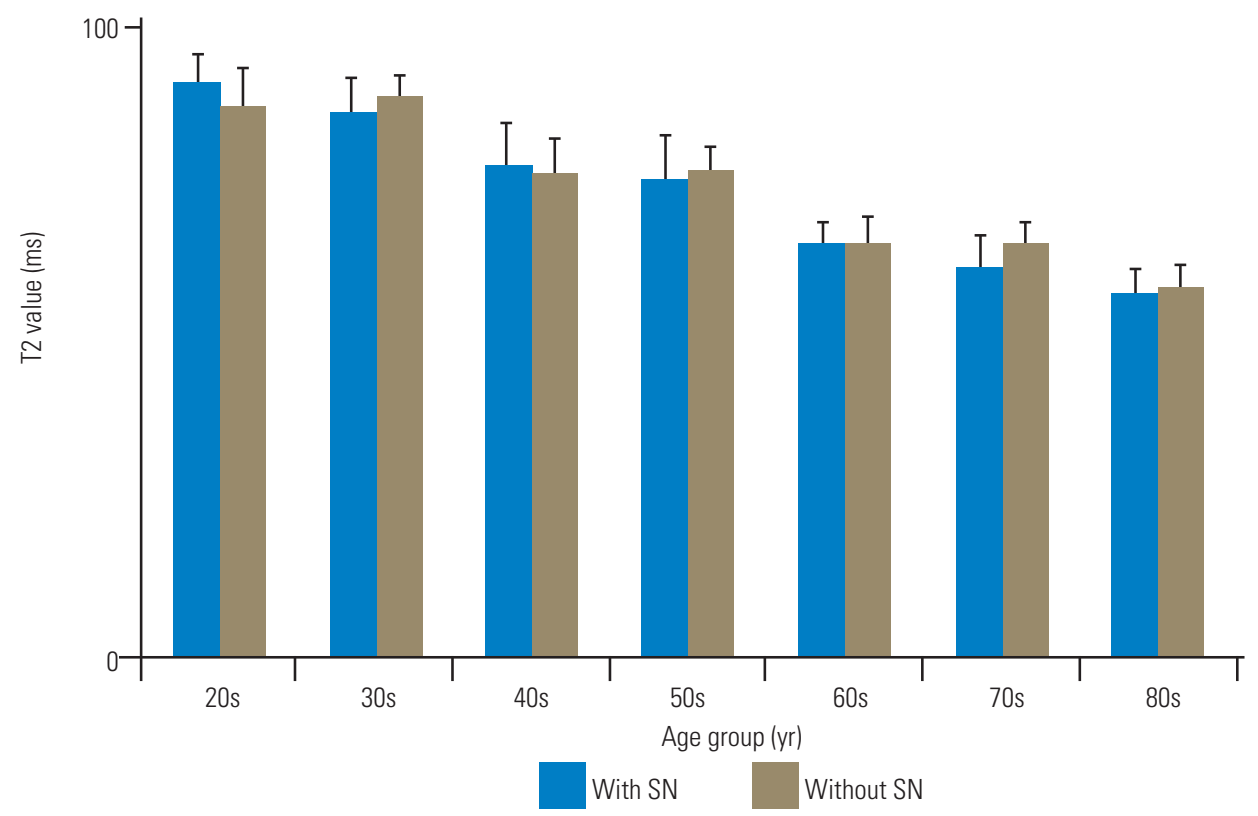

Fig. 6. Bar chart of T2 values with and without SN as NP. There were no remarkable differences in the T2 values of NP per the age decade. Error bars denote the standard error of the mean values. SN, Schmorl's node; NP, nucleus pulposus. 


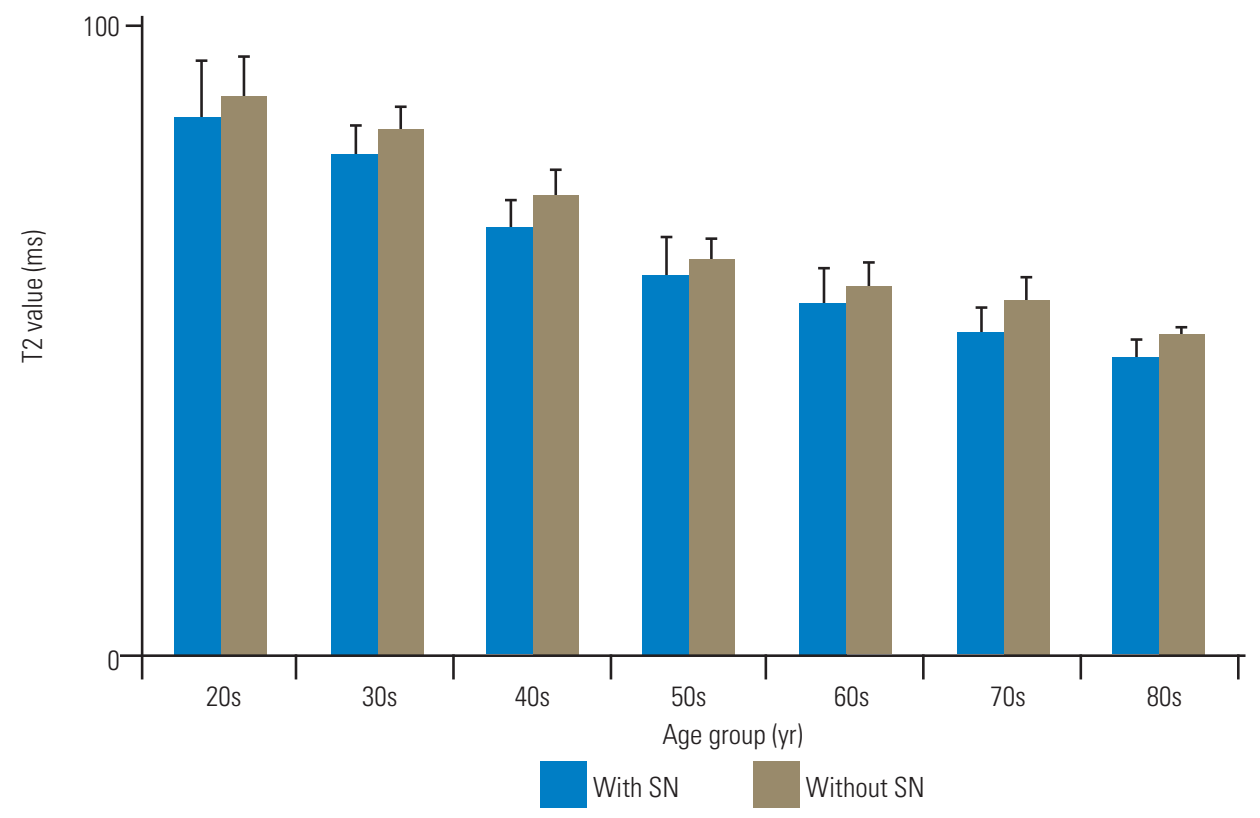

Fig. 7. Bar chart of the $T 2$ values with and without SN as posterior $A F$. There were no remarkable differences in the $T 2$ values of posterior AF as per the age decade. Error bars denote the standard error of the mean values. SN, Schmorl's node; AF, annulus fibrosus.

80s (Fig. 6). There were no significant differences in the $\mathrm{T} 2$ values of NP as per the age decade $(p=0.68,0.65,0.82$, $0.70,0.81,0.61$, and 0.72 , respectively). Moreover, the T2 values of the posterior AF with and without $\mathrm{SN}$ were as follows, respectively: $86.1 \pm 9.6 \mathrm{~ms}$ and $89.7 \pm 6.1 \mathrm{~ms}$ in the $20 \mathrm{~s} ; 80.3 \pm 4.6 \mathrm{~ms}$ and $84.1 \pm 4.1 \mathrm{~ms}$ in the $30 \mathrm{~s}, 68.1 \pm 5.2$ $\mathrm{ms}$ and $73.9 \pm 3.8 \mathrm{~ms}$ in the $40 \mathrm{~s} ; 60.9 \pm 6.5 \mathrm{~ms}$ and $63.2 \pm 3.7$ $\mathrm{ms}$ in the $50 \mathrm{~s} ; 56.2 \pm 5.9 \mathrm{~ms}$ and $59.1 \pm 4.0 \mathrm{~ms}$ in the $60 \mathrm{~s}$; $51.9 \pm 4.0 \mathrm{~ms}$ and $57.0 \pm 3.9 \mathrm{~ms}$ in the $70 \mathrm{~s}$; and $47.6 \pm 3.2 \mathrm{~ms}$ and $51.6 \pm 1.3 \mathrm{~ms}$ in the 80 s (Fig. 7). There were no significant differences in the $\mathrm{T} 2$ values of posterior $\mathrm{AF}$ as per the age decade $(p=0.71,0.61,0.42,0.67,0.74,0.46$, and 0.50 , respectively).

\section{Discussion}

In this study, a total of $30.5 \%$ of the subjects presented with SNs, with prevalence $(70.3 \%)$ in the upper 2 lumbar levels (L1/2 and L2/3). This finding was consistent with that of the previous reports, which showed that SNs were more commonly located in the thoracolumbar region $[6,16,17]$. This result could be attributed to the higher mechanical stresses at the transition zone between the thoracic and lumbar spine. It has also been reported that compressive loading on the posterior facet ligamentous complex of the lumbar spine increased with a rise in lor- dotic posture [18] and that the average loading on the facet joints of the three lowest lumbar segments was higher than that of the two upper segments [19]. Therefore, with the load sharing between the IVD and the paired facets, one would postulate that the relative compressive forces loaded onto the IVD should decrease caudally owing to the increase in lumbar lordosis. This effect, in turn, may account for the lower compressive forces loaded onto the endplate, which results in less $\mathrm{SN}$ presence on the lower lumbar segments. Moreover, a recent skeletal study, which examined endplate strength with the indentation loading test, found that there were remarkable differences between the lumbar segments in both the superior and inferior endplates and that the mechanical strength tended to increase caudally. This suggested that the endplates of the upper lumbar segments were weaker than those of the lower lumbar segments [20]. In addition, these findings indicate the importance of endplate strength in SN etiology. Furthermore, SN occurrence was reported because of such factor as traumatic history during childhood, genetic involvement [18,21], and so on. The effect of cumulative minor injuries, such as compressive axial loading, might be an underlying mechanism that could result in endplate damage and eventually SN development. Moreover, it was noted that SNs were strongly determined genetically with heritability as high as $72 \%$ and $80 \%$ over the thoracic and 
lumbar spine, respectively, in a study with twin subjects [18]. Therefore, we considered that the percent prevalence of SN did not increase with older age.

We also elucidated the association between IVDD and SN in CLBP with quantitative MRI and found that the T2 values of the lumbar discs nearly decreased with age and $\mathrm{T} 2$ values of anterior $\mathrm{AF}$ in subjects with $\mathrm{SN} \geq 50$ years were remarkably lower than those without SN. Several studies have revealed an association between SN and IVDD $[6,16,18,22,23]$. A cadaveric study reported that the pressure in the IVD could be reduced by $25 \% \pm 27 \%$ with minor endplate damage [24]. Similarly, endplate fracture produced an immediate effect by remarkable reduction of the IVD pressure to $37 \%$ in cadaveric human lumbar discs [25]. We considered that SN converted stresses and loads against IVD and shared a common pathway with anterior AF degeneration. Moreover, this study found that the presence of $\mathrm{SN}$ is not a risk factor for CLBP itself; however, it is an indicator of IVDD of anterior AF. There were agreements on the lack of association between SN and CLBP, including the relation between SN and IVDD in past reports $[16,18,26]$. An interesting finding of this study was the identification of the part of IVDD, that is, anterior AF, quantitatively relative to $\mathrm{SN}$.

Previously, we evaluated the extent of IVDD and compared this with the T2 values in groups with degenerative spondylolisthesis (DS) and no spondylolisthesis. We found that the $\mathrm{T} 2$ values decreased in the anterior $\mathrm{AF}$ of IVD in the DS group [27]. In this regard, characterization of the relationship between degeneration in the anterior $\mathrm{AF}$ and $\mathrm{SN}$ may result in an accurate noninvasive evaluation and subsequent treatment and surgical planning, which would be especially useful when fusion surgery is needed. Our study also suggested the possibility of early diagnosis of $\mathrm{SN}$ degeneration and expected prediction of adjacent segmental disease after posterior spinal fusion $[28,29]$.

This study has certain limitations. First, the crosssectional study design limited the results regarding timecourse of the association between SN and IVDD. Longitudinal studies would be necessary for detailed analysis. Second, we did not evaluate the SN characteristics, such as location, diameter, and volume. Third, the number of subjects with SN was too small to confirm or refute an association, and there were no control cases. We should analyze the cases without LBP and with SN and investigate on their treatment in the future.
In summary, there were no remarkable age decade- or gender-based differences in the prevalence rate of SNs. Furthermore, SNs were prevalent in the upper 2 lumbar levels (L1/2 and L2/3). The presence of SNs is not itself a risk factor for CLBP; however, it indicates IVDD of the anterior AF in subjects with $\mathrm{SN}$ who are $\geq 50$ years old.

\section{Conflict of Interest}

No potential conflict of interest relevant to this article was reported.

\section{References}

1. Deyo RA, Weinstein JN. Low back pain. N Engl J Med 2001;344:363-70.

2. Hurri H, Karppinen J. Discogenic pain. Pain 2004;112:225-8.

3. Kyere KA, Than KD, Wang AC, et al. Schmorl's nodes. Eur Spine J 2012;21:2115-21.

4. Gonzalez-Reimers E, Mas-Pascual M, Arnay-De-LaRosa M, Velasco-Vazquez J, Santolaria-Fernandez F. Schmorl nodes: lack of relationship between degenerative changes and osteopenia. Radiology 2002;222:293-4.

5. Takahashi K, Miyazaki T, Ohnari H, Takino T, Tomita K. Schmorl's nodes and low-back pain: analysis of magnetic resonance imaging findings in symptomatic and asymptomatic individuals. Eur Spine J 1995;4:569.

6. Pfirrmann CW, Resnick D. Schmorl nodes of the thoracic and lumbar spine: radiographic-pathologic study of prevalence, characterization, and correlation with degenerative changes of 1,650 spinal levels in 100 cadavers. Radiology 2001;219:368-74.

7. Zou J, Yang H, Miyazaki M, et al. Dynamic bulging of intervertebral discs in the degenerative lumbar spine. Spine (Phila Pa 1976) 2009;34:2545-50.

8. Pfirrmann CW, Metzdorf A, Zanetti M, Hodler J, Boos N. Magnetic resonance classification of lumbar intervertebral disc degeneration. Spine (Phila $\mathrm{Pa}$ 1976) 2001;26:1873-8.

9. Karakida O, Ueda H, Ueda M, Miyasaka T. Diurnal $\mathrm{T} 2$ value changes in the lumbar intervertebral discs. Clin Radiol 2003;58:389-92.

10. Krueger EC, Perry JO, Wu Y, Haughton VM. Changes in T2 relaxation times associated with maturation of 
the human intervertebral disk. AJNR Am J Neuroradiol 2007;28:1237-41.

11. Marinelli NL, Haughton VM, Anderson PA. T2 relaxation times correlated with stage of lumbar intervertebral disk degeneration and patient age. AJNR Am J Neuroradiol 2010;31:1278-82.

12. Perry J, Haughton V, Anderson PA, Wu Y, Fine J, Mistretta $C$. The value of $\mathrm{T} 2$ relaxation times to characterize lumbar intervertebral disks: preliminary results. AJNR Am J Neuroradiol 2006;27:337-42.

13. Zuo J, Joseph GB, Li X, et al. In vivo intervertebral disc characterization using magnetic resonance spectroscopy and T1 $\rho$ imaging: association with discography and Oswestry Disability Index and Short Form36 Health Survey. Spine (Phila Pa 1976) 2012;37:21421.

14. Takashima H, Takebayashi T, Yoshimoto M, et al. Correlation between $\mathrm{T} 2$ relaxation time and intervertebral disk degeneration. Skeletal Radiol 2012;41:1637.

15. Ogon I, Takebayashi T, Takashima H, et al. Analysis of chronic low back pain with magnetic resonance imaging T2 mapping of lumbar intervertebral disc. J Orthop Sci 2015;20:295-301.

16. Mok FP, Samartzis D, Karppinen J, Luk KD, Fong DY, Cheung KM. ISSLS prize winner: prevalence, determinants, and association of Schmorl nodes of the lumbar spine with disc degeneration: a populationbased study of 2449 individuals. Spine (Phila Pa 1976) 2010;35:1944-52.

17. Stabler A, Bellan M, Weiss M, Gartner C, Brossmann J, Reiser MF. MR imaging of enhancing intraosseous disk herniation (Schmorl's nodes). AJR Am J Roentgenol 1997;168:933-8.

18. Williams FM, Manek NJ, Sambrook PN, Spector TD, Macgregor AJ. Schmorl's nodes: common, highly heritable, and related to lumbar disc disease. Arthritis Rheum 2007;57:855-60.

19. Adams MA, Hutton WC. The effect of posture on the role of the apophyseal joints in resisting intervertebral compressive forces. J Bone Joint Surg Br 1980;62:358-62.
20. Hou Y, Luo Z. A study on the structural properties of the lumbar endplate: histological structure, the effect of bone density, and spinal level. Spine (Phila Pa 1976) 2009;34:E427-33.

21. Moller A, Maly P, Besjakov J, Hasserius R, Ohlin A, Karlsson MK. A vertebral fracture in childhood is not a risk factor for disc degeneration but for Schmorl's nodes: a mean 40-year observational study. Spine (Phila Pa 1976) 2007;32:2487-92.

22. Hilton RC, Ball J, Benn RT. Vertebral end-plate lesions (Schmorl's nodes) in the dorsolumbar spine. Ann Rheum Dis 1976;35:127-32.

23. Wu HT, Morrison WB, Schweitzer ME. Edematous Schmorl's nodes on thoracolumbar MR imaging: characteristic patterns and changes over time. Skeletal Radiol 2006;35:212-9.

24. Adams MA, Freeman BJ, Morrison HP, Nelson IW, Dolan P. Mechanical initiation of intervertebral disc degeneration. Spine (Phila Pa 1976) 2000;25:1625-36.

25. Przybyla A, Pollintine P, Bedzinski R, Adams MA. Outer annulus tears have less effect than endplate fracture on stress distributions inside intervertebral discs: relevance to disc degeneration. Clin Biomech (Bristol, Avon) 2006;21:1013-9.

26. Kjaer P, Leboeuf-Yde C, Korsholm L, Sorensen JS, Bendix T. Magnetic resonance imaging and low back pain in adults: a diagnostic imaging study of 40-year-old men and women. Spine (Phila Pa 1976) 2005;30:1173-80.

27. Takashima H, Takebayashi T, Yoshimoto M, et al. Investigation of intervertebral disc and facet joint in lumbar spondylolisthesis using T2 mapping. Magn Reson Med Sci 2014;13:261-6.

28. Akamaru T, Kawahara N, Tim Yoon S, et al. Adjacent segment motion after a simulated lumbar fusion in different sagittal alignments: a biomechanical analysis. Spine (Phila Pa 1976) 2003;28:1560-6.

29. Pellise F, Hernandez A, Vidal X, Minguell J, Martinez C, Villanueva C. Radiologic assessment of all unfused lumbar segments 7.5 years after instrumented posterior spinal fusion. Spine (Phila Pa 1976) 2007;32:5749. 